\title{
Los saberes a través de la lectura
}

\section{Potentializing knowledge through reading}

\author{
Juana Isabel Rodríguez Bach \\ Facultad de Humanidades \\ Universidad de San Carlos de Guatemala \\ https://orcid.org/0000-0002-8874-5183 \\ isarodriguez1607@gmail.com
}

\section{Referencia}

Rodríguez Bach, J. I. (2021). Los Saberes a través de la lectura. Revista Guatemalteca de Educación Superior, 4(1), pp 59-71. DOI: https://doi.org/10.46954/revistages.v4i1.54

Recibido: 15/11/2020

Aceptado: 16/01/2021

\section{Resumen}

Este artículo se fundamenta en la importancia de las estrategias de lectura y su influencia en la comprensión lectora, teniendo en cuenta que la comprensión lectora es uno de las destrezas que los estudiantes deben tener cuando ingresan a la educación superior. Por lo que da a conocer los resultados de la investigación que tuvo por objetivo: demostrar la influencia de las estrategias de lectura en la mejora de la comprensión lectora de los estudiantes de segundo semestre, plan sabatino del profesorado de Lengua y Literatura, EFPEM. Tiene importancia porque presenta de forma descriptiva la incidencia de las estrategias de lectura con la comprensión lectora, para coadyuvar el nivel de comprensión lectora de los estudiantes universitarios.

Este se realizó con una población de 37 mujeres y 4 hombres; para el efecto se ejecutó una investigación cuantitativa, a través de un estudio experimental realizado un pretest el cual evidenció las estrategias empleadas por los estudiantes y el 
nivel de comprensión lectora, posteriormente se intervino con cuatro talleres, después de la intervención se aplicó un postest, donde se obtuvo las conclusiones: Las estrategias de lectura tienen influencia con la mejora de la comprensión lectora de los estudiantes que fueron sujetos de dicho estudio. Las estrategias de lectura empleadas son: antes de la lectura; establecen el propósito de la lectura; durante la lectura, monitorean su propia

Palabras clave:

educación superior, lectura, estrategias de lectura, niveles de comprensión lectora comprensión; después de la lectura los estudiantes realizan resúmenes. El nivel de comprensión lectora de los estudiantes después de la intervención mejoró en los tres niveles.

\section{Abstract}

This article is based on the importance of reading strategies and their influence on reading comprehension, taking into account that reading comprehension is one of the skills that students must have when they enter higher education. Therefore, the article reveals the results of the following objective: Demonstrate the influence of reading strategies in improving the reading comprehension of second semester students, Saturday plan of the teachers of Language and Literature, EFPEM. It is important because it presents in a descriptive way the incidence of reading strategies with reading comprehension, to contribute to the level of reading comprehension of university students. This was carried out with a population of 37 women and 4 men; For this purpose, a quantitative research was carried out, through an experimental study carried out a pretest which evidenced the strategies used by the students and the level of reading comprehension, later it was intervened with four workshops, after the intervention a posttest was applied, where the conclusions were obtained: The reading strategies have an influence with the improvement of the reading comprehension of the students who were the subjects of said study. The reading strategies used are: before reading;

\section{Keywords:}

higher education, reading, reading strategies, levels of reading comprehension. establish the purpose of the reading; during reading, they monitor their own comprehension; after reading the students make summaries. The level of reading comprehension of the students after the intervention improved in all three levels. 


\section{Introducción}

Este artículo se ha elaborado con un tema de interés académico, laboral, y social por ello se ha titulado"Los Saberes a Través de la Lectura, puesto que la comprensión lectora es una habilidad básica sobre la cual se despliega una serie de capacidades: la empatía, el manejo de la oralidad y pensamiento crítico. Sin embargo los estudiantes del segundo semestre del profesorado de Lengua y Literatura, de la Escuela de Formación de Profesores de Enseñanza Media presentaron dificultades para comprender lo que leen, no siguieron instrucciones textuales del material de apoyo, leen solo superficialmente; falta de interés por la lectura, la escasa capacidad analítica, bajo nivel de comprensión; pobre desarrollo de la competencia comunicativa. Esto conlleva a la dificultad de apropiarse del conocimiento por cuenta propia; ausencia de un pensamiento lógico y crítico, por lo que presentan tareas con escasa información requerida; aunado a ello, la pobreza léxica contribuye al problema que tienen para identificar el sentido concreto de ciertos términos.

Este tiene importancia científica dado que se evalúa y analiza lineamientos teóricos científicos para presentar de forma descriptiva la incidencia de las estrategias de lectura que emplean los estudiantes del Profesorado de Lengua y Literatura, de la Escuela de Formación de Profesores de Enseñanza Media, con el nivel de la comprensión lectora, en cuanto a la aplicación de actividades pedagógicas alternativas para mejorar las estrategias de comprensión de textos escritos. Se justifica también desde un punto de vista teleológico, porque da cuenta el papel primordial del docente, en ser moderador y facilitador de las estrategias pues éstas deben ser enseñadas. Así mismo el tema está orientado a potencializar las habilidades y capacidades comunicativas de los universitarios en relación a las estrategias de metacomprensión. Por lo que tuvo como objetivo general. Demostrar la influencia de las estrategias de lectura en la mejora de la comprensión lectora de los estudiantes de segundo semestre, plan sabatino del profesorado de Lengua y Literatura, de la Escuela de Formación de Profesores de Enseñanza Media, y como objetivos específicos: Identificar las estrategias de lectura que emplean los estudiantes y determinar el nivel de comprensión lectora de los estudiantes del segundo semestre de dicha escuela de la universidad de San Carlos de Guatemala. 


\section{Materiales y métodos}

La investigación realizada tuvo un enfoque cuantitativo; el alcance fue de tipo descriptivo, con un diseño experimental ya que se aplicó a los sujetos de estudios una prueba pre y post test, para establecer la influencia de las estrategias de lectura en el nivel de comprensión lectora. Desde el horizonte temporal, fue un estudio longitudinal que permitió obtener datos en dos momentos diferentes. Se tuvo una población de 190 estudiantes del segundo semestre del profesorado plan sabatino, de Lengua y Literatura, del 2020 solamente 41 participaron en el pre test, pos test y en las actividades de la intervención, la muestra fue tomada por conveniencia. Del total $10 \%$ son hombres y el $90 \%$ mujeres, están comprendidos en las edades 21-49.

Para el sustento teórico científico se da a conocer algunas definiciones: "la comprensión lectora hace referencia a un proceso simultaneo de extracción y construcción transaccional entre las experiencias y conocimientos del lector, con el texto escrito en un contexto de actividad" Ronsenblatt, Hoyos. y Gallego (2017). De lo definido por el autor la comprensión es un proceso dinámico que permite esa interacción del texto con el contexto, logrando así un aprendizaje significativo. Para lograr este cometido es necesario valerse de estrategias; estas son definidas como herramientas del docente para contribuir al desarrollo de las competencias de los estudiantes. Pimienta (2012 p3). Como lo define Pimienta es necesario contar con herramientas de aprendizaje para desarrollar, habilidades y destrezas de aprendizaje, esas herramientas de pensamiento son los que, coordinan y aplican las habilidades que logran el aprendizaje significativo y con el "aprender a aprender". Como lo define el autor estas herramientas estimulan diferentes procesos cognitivos, que contribuyen a mejorar las competencias que permiten encontrar significatividad a lo aprendido, permitiendo dar una dirección hacia donde encaminar el aprendizaje.

Se utilizó una prueba estandarizada que fue validada por medio de una prueba piloto por personal de La Dirección General de Calidad Educativa -DIGEDUCA- El cuestionario en escala de likert 
fue validada a Juicio de expertos. Tres docentes de la Maestría en Docencia de la Educación Superior con experiencia en este campo. Para el análisis de resultados se realizó una base de datos en un archivo Excel, para conocer los resultados de los instrumentos del pre test tanto del nivel de comprensión lectora, como de las estrategias utilizadas por los estudiantes. A partir de los resultados obtenidos se planteó la intervención de 4 talleres en distintos momentos para conocer sí las estrategias tienen influencia en la comprensión lectora. Estos talleres se llevaron a cabo por medio de video conferencias a través de la plataforma Zoom, con una duración de 45 minutos y ejercicios de práctica asincrónica.

\section{Resultados}

Presentación de resultados de la aplicación del pre y post test de una prueba estandarizada de comprensión lectora del Ministerio de Educación de Guatemala. Esta es una prueba liberada, GRAD-A se encuentra en el portal de la página del Ministerio de Educación de Guatemala. Aplicado a los estudiantes del segundo semestre del profesorado de Lengua y Literatura plan sabatino 2020, en los meses de agosto y octubre del 2020.

Tabla No. 1 resumen comparativo entre el pretest y postest

\begin{tabular}{|c|c|c|c|c|c|c|}
\hline & & & Pre-test & & Post-test & \\
\hline N0. & Preguntas & No. & Correctas & Incorrectas & Correctas & Incorrectas \\
\hline 1 & $\begin{array}{l}\text { ¿Cuál de las siguientes palabras es un } \\
\text { antónimo de erguido? }\end{array}$ & 1 & 13 & 28 & 30 & 11 \\
\hline 2 & $\begin{array}{l}\text { ¿Cuál de las siguientes palabras es un } \\
\text { sinónimo de incrementar? }\end{array}$ & 2 & 15 & 26 & 22 & 19 \\
\hline 3 & $\begin{array}{l}\text { Clara sintió una enorme tristeza a } \\
\text { consecuencia de }\end{array}$ & 3 & 18 & 23 & 38 & 3 \\
\hline 4 & $\begin{array}{l}\text { ¿qué planta de piña tiene sus hojas con } \\
\text { machas rojizas y bordes } \\
\text { lisos? }\end{array}$ & 4 & 15 & 26 & 32 & 9 \\
\hline
\end{tabular}




\begin{tabular}{|c|c|c|c|c|c|c|}
\hline 5 & $\begin{array}{l}\text { ¿qué similitud hay entre los tipos de } \\
\text { piña? }\end{array}$ & 5 & 12 & 29 & 30 & 11 \\
\hline 6 & $\begin{array}{l}\text { ¿cuál es el orden para preparar caldo de } \\
\text { gallina? }\end{array}$ & 6 & 17 & 24 & 28 & 13 \\
\hline 7 & ¿cuál es la idea principal? & 7 & 13 & 28 & 28 & 13 \\
\hline 8 & $\begin{array}{l}\text { ¿Cuál es el orden en el que sucedieron } \\
\text { los acontecimientos? }\end{array}$ & 8 & 11 & 30 & 30 & 11 \\
\hline 9 & $\begin{array}{l}\text { Según el texto, la palabra albergar } \\
\text { significa... }\end{array}$ & 9 & 13 & 28 & 22 & 19 \\
\hline 10 & $\begin{array}{l}\text { ¿Cuál de las siguientes opciones } \\
\text { corresponde a la intención } \\
\text { comunicativa del texto? }\end{array}$ & 10 & 16 & 25 & 38 & 3 \\
\hline 11 & $\begin{array}{l}\text { ¿qué podría suceder a causa de la nube } \\
\text { de polvo? }\end{array}$ & 11 & 10 & 31 & 26 & 15 \\
\hline 12 & $\begin{array}{l}\text { ¿Cuál de las siguientes opciones } \\
\text { corresponde a la intención } \\
\text { comunicativa del texto? }\end{array}$ & 12 & 16 & 25 & 29 & 12 \\
\hline 13 & $\begin{array}{l}\text { ¿Cuál palabra significa lo contrario de } \\
\text { convergen? }\end{array}$ & 13 & 19 & 22 & 30 & 11 \\
\hline 14 & $\begin{array}{l}\text { ¿Cuál de los siguientes enunciados es } \\
\text { un hecho que aparece en } \\
\text { El }\end{array}$ & 14 & 16 & 25 & 23 & 18 \\
\hline 15 & $\begin{array}{l}\text { Según el texto, la palabra polígrafo } \\
\text { significa }\end{array}$ & 15 & 14 & 27 & 28 & 13 \\
\hline 16 & $\begin{array}{l}\text { ¿Cuál de las siguientes opciones es el } \\
\text { propósito del texto? }\end{array}$ & 16 & 15 & 26 & 24 & 17 \\
\hline 17 & ¿De qué trata el texto? & 17 & 18 & 23 & 38 & 3 \\
\hline 18 & $\begin{array}{l}\text { ¿Qué utiliza este animal para } \\
\text { desplazarse? }\end{array}$ & 18 & 19 & 22 & 23 & 18 \\
\hline 19 & $\begin{array}{l}\text { Según la información del texto, por lo } \\
\text { general, una de las } \\
\text { superficies de las lentes convergentes } \\
\text { son de superficie... }\end{array}$ & 19 & 12 & 29 & 23 & 18 \\
\hline \multirow[t]{2}{*}{20} & ¿Cuál es la idea principal del texto? & 20 & 11 & 30 & 24 & 17 \\
\hline & Promedio & & 15 & 26 & 28 & 13 \\
\hline
\end{tabular}

Fuente propia, tomada de la prueba pre y post de la lectura aplicada a estudiantes del profesorado de Lengua y Literatura plan sabatino durante (2020) de la Escuela de Profesores de Enseñanza Media 
En No. 1 muestra los resultados obtenidos por cada pregunta, brindada por los estudiantes que conforman la muestra correspondiente pretest y postest las preguntas que corresponden al nivel literal son: 1,2,4, 6, 8, 13, 14,17, 18; el nivel inferencial fue evaluado a través de los siguientes ítems $3,5,7,9,11,15,19$ y 20 y el para verificar el nivel crítico se $10,12,16$. Donde se hace una comparación de los resultados, afirmando la importancia de la enseñanza de las estrategias de lectura para mejorar la comprensión lectora ya que en el estudio realizado evidencian mejoras.

Figura No. 1 Resumen comparativo entre el pretest y postest

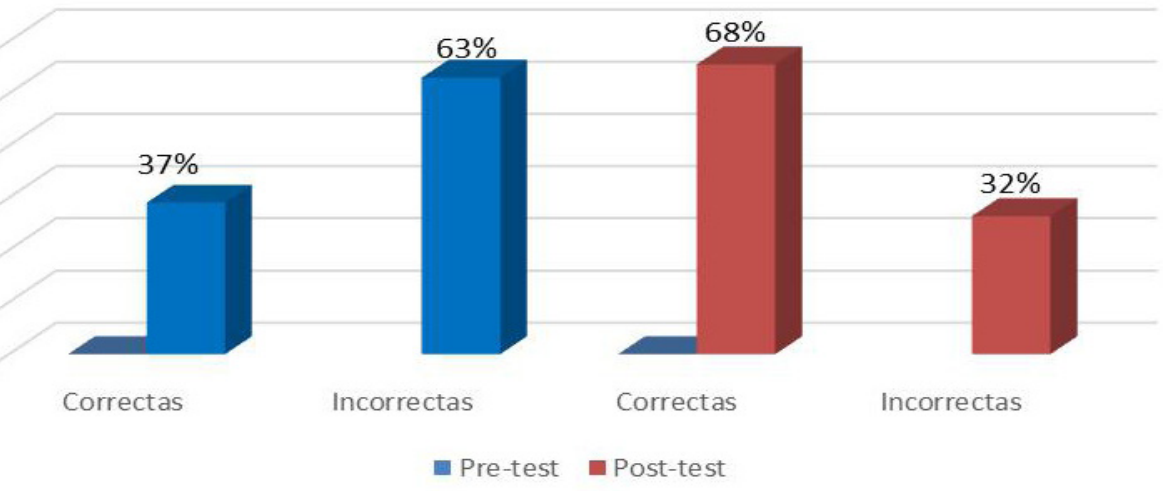

Fuente propia, tomada de la prueba pre y post de la lectura aplicada a estudiantes de profesorado de Lengua y Literatura plan sabatino durante (2020) de la Escuela de Profesores de Enseñanza Media.

El figura anterior muestra los resultados obtenidos por cada pregunta, brindada por los estudiantes que conforman la muestra correspondiente pretest y postest. Donde se evidencia la mejora de la comprensión, por lo que es importante trabajar el proceso lector para mejorar la compresión lectora.

Tabla No. 2 Resumen del nivel de comprensión lectora 'pre y post test que fue de 20 preguntas resueltas por 41 estudiantes, dando un resultado de 820 respuestas, se muestra la distribución según e nivel de comprensión en la siguiente tabla. 


\begin{tabular}{|l|l|l|l|l|}
\hline Preguntas & Pre test & & post test & \\
\hline & Correctas & Incorrectas & Correctas & Incorrectas \\
\hline Literales & 153 & 257 & 282 & 128 \\
\hline Inferenciales & 68 & 137 & 141 & 64 \\
\hline Críticas & 20 & 103 & 91 & 32 \\
\hline Total & 293 & 527 & 566 & 254 \\
\hline Preguntas & Pre test & & post test & \\
\hline Lríticas & $16 \%$ & $84 \%$ & $91 \%$ & $32 \%$ \\
\hline Literales & $37 \%$ & $63 \%$ & $69 \%$ & $31 \%$ \\
\hline Inferenciales & $33 \%$ & $67 \%$ & $69 \%$ & $31 \%$ \\
\hline & & & & \\
\hline
\end{tabular}

Fuente propia, tomada de la prueba pre y post de la lectura aplicada a estudiantes del profesorado de Lengua y Literatura plan sabatino durante (2020) de la Escuela de Profesores de Enseñanza Media.

La tabla No. 2 muestra el resumen de los resultados obtenidos por cada pregunta, relacionada a los distintos niveles de comprensión lectora tanto del pretest y postest, donde se reflejó que previo a la intervención con talleres de comprensión lectora donde se aplicó el proceso lector, el nivel literal, inferencial y crítico estuvo por debajo de la media, pero después de la medición mejoró los tres niveles. 
Tabla No. 3 Resultados en frecuencias absolutas de las estrategias utilizadas por estudiantes

\begin{tabular}{|c|c|c|c|c|}
\hline Característica & Nunca & $\begin{array}{l}\text { Pocas } \\
\text { veces }\end{array}$ & Siempre & TOTAL \\
\hline $\begin{array}{l}\text { 1) Activa sus conocimientos previos } \\
\text { relacionando lo conocido con lo observado } \\
\text { en el libro. }\end{array}$ & 0 & 16 & 25 & 41 \\
\hline $\begin{array}{l}\text { 2) Establece un propósito antes de realizar } \\
\text { la lectura del texto }\end{array}$ & 0 & 7 & 34 & 41 \\
\hline $\begin{array}{l}\text { 3) Realiza hipótesis sobre lo que ocurrirá en } \\
\text { el texto, tomando en: el título, ilustraciones, } \\
\text { además de las experiencias y conocimiento } \\
\text { propios }\end{array}$ & 0 & 28 & 12 & 41 \\
\hline $\begin{array}{l}\text { 4) Monitorea su comprensión, estando alerta } \\
\text { para determinar si se está comprendiendo } \\
\text { en todo momento y si no es así, interrumpe } \\
\text { la lectura para poder reparar la comprensión }\end{array}$ & 1 & 12 & 28 & 41 \\
\hline $\begin{array}{l}\text { 5) Decodifica el lenguaje figurado mientras } \\
\text { le el texto }\end{array}$ & 1 & 27 & 13 & 41 \\
\hline $\begin{array}{l}\text { 6) Durante la lectura comparar las hipótesis } \\
\text { y predicciones que se hacen antes de leer, } \\
\text { con lo que el texto expresa. }\end{array}$ & 3 & 26 & 12 & 41 \\
\hline $\begin{array}{l}\text { 7) Mientras lee conecta los temas } \\
\text { ahilándolos entre sí. }\end{array}$ & 1 & 15 & 25 & 41 \\
\hline $\begin{array}{l}\text { 8) Después de leer realiza conexiones } \\
\text { relacionando lo que ocurre en el texto con } \\
\text { su propia vida, con otro texto y con su } \\
\text { conocimiento sobre el mundo. }\end{array}$ & 41 & 0 & 0 & 41 \\
\hline $\begin{array}{l}\text { 9) Expresar lo que piensa, acuerdos y } \\
\text { desacuerdos con el texto, con las ideas } \\
\text { explícitas e implícitas, con las propuestas } \\
\text { presentadas y sus consecuencias }\end{array}$ & 1 & 26 & 14 & 41 \\
\hline $\begin{array}{l}\text { 10) Identifica la información más relevante, } \\
\text { establece relaciones entre ideas principales } \\
\text { esquematizándolas en una estructura, marco } \\
\text { de referencia }\end{array}$ & 2 & 22 & 17 & 41 \\
\hline
\end{tabular}

Fuente propia, tomada de cuestionario en escala de Likert relacionado a las estrategias de comprensión lectora, aplicado a estudiantes del profesorado de Lengua y Literatura plan sabatino durante (2020) de la Escuela de Profesores de Enseñanza Media. 
En la tabla No. 3 muestra los resultados obtenidos por cada pregunta, relacionada a las estrategias de lectura que emplean los estudiantes, donde se evidencia que utilizan más estrategias antes de la lectura, entre los que sobre salen: establecen el propósito de la lectura y establecen la hipótesis de lo que ocurrirá en el texto; durante la lectura muy pocos estudiantes decodifican el lenguaje figurado y después de la únicamente realizan resúmenes las otras estrategias mencionas en el test como realizar conexiones con otras áreas curriculares, relacionar el texto con su contexto y su vida en su totalidad no lo utilizan. Al no utilizar el proceso lector (antes durante y después) tiene como consecuencia la baja comprensión lectora.

\section{Discusión}

De acuerdo los resultados obtenidos del pre test de la prueba estandarizada del nivel de comprensión lectora del Ministerio de Educación, Guatemala, los estudiantes de segundo semestre, del profesorado de Lengua y Literatura, plan sabatino de la Escuela de Formación de Profesores de Enseñanza Media durante 2020, se les dificultó localizar la idea principal, identificar la intención comunicativa, relación de causa y efecto en los textos presentados, de esta cuenta se afirma que los estudiantes no establecieron un análisis de los textos que permitieran establecer la relación de lógica, así mismo confunden las ideas principales con las secundaria. Esto no coincide con lo que afirman más de $35 \%$ de estudiantes en utilizar estrategias: antes, durante y después de la lectura, por lo que se infiere que no tienen claro el manejo de las estrategias. Ya que solo el 37\% logran el nivel inferencial. Este tiene estrecha relación con la teoría de Márquez (2016) donde afirma que los estudiantes poseen un adecuado nivel de comprensión literal, se centra en las ideas e información que están explícitamente expuestas en el texto por reconocimiento o evocación de hechos.

Después de la intervención que consistió en cuatro talleres en Zoom con una duración de 45 minutos y las estrategias que se abordaron fueron las siguientes: taller No. 1 Identificación de ideas principales, la tipología de texto utilizado fue Informativo, objetivo: Identificar ideas principales explicitas en textos informativos y su función es facilitar la comprensión de un 
acontecimiento al lector. Para ello, incluye hechos, descripciones y datos, el taller No.2 Identificación de hilo conductor, tipología de texto; literario, objetivo, Identificar el hilo conductor, taller No. 3 la estrategia, establecer relaciones, tipología de texto, informativo de tipo literaria y el taller No. 4 cómo relacionar la lectura con el contexto y otras áreas curriculares, tipología de texto, literario, objetivo, relacionar la lectura con el contexto y otras áreas curriculares para generar nuevos aprendizajes desde la lectura.

Después de esta intervención se procedió a aplicar el pos test donde se reflejó en los resultados una notable mejora en la comprensión lectora esto se evidencia en las respuestas correctas de las preguntas en los distintos niveles. Aunque no se alcanzó el $100 \%$ de mejora por el tiempo que fue muy corto, cabe resaltar que enseñando estrategias de lectura los estudiantes pueden mejorar su nivel de comprensión lectora, así también lo afirma Solé (1998) "las estrategias deben de ser enseñadas y mediadas por el docente" ya que los estudiantes por si solos no logran realizar los procesos correctamente, pero al darles un acompañamiento son capaces de recrear las estrategias con otros textos de su interés y reflejar en su trabajo académico un grado de análisis más profundo, está es una de las razones de formar lectores, capaces de enfrentarse de manera inteligente a textos de muy distinta índole. Por esta razón es importante enseñar estrategias para contribuir con un aprendizaje integral, esto concuerda con lo que dicen Roncal y Montepeque (2011) de la importancia de usar estrategias de comprensión lectora, ya que estos contribuyen a lograr la comprensión lectora y favorecen el aprendizaje significativo, ellos afirman que las estrategias lectoras contribuyen a formar lectores autónomos, porque dichas estrategias les orienta, a cuestionar sobre los que están aprendiendo con el texto. Logros: a. Con las estrategias lectoras trabajadas en los talleres, aumentó el porcentaje de comprensión en el nivel literal, inferencial y crítico. b. Trabajar las estrategias de lectura desde la interdisciplinariedad permitió el logro de capacidades de análisis y síntesis. C. A través de los talleres los estudiantes adquirieron un conjunto de herramientas didácticas de lectura para aplicar en diferentes textos y contextos. 


\section{Agradecimientos}

A la Dra. María lliana Cardona de Chavac, por aceptar asesorarme en proceso de redacción de este artículo, a pesar de sus múltiples compromisos, siempre tuvo un espacio para solventar mis dudas, alentarme a seguir adelante y guiarme en el camino de la investigación. A mi docente de investigación: Bidel Méndez, por la confianza y conocimientos que me ha permitido avanzar y aprender diferentes procesos de la investigación. Al comité de la Revista Guatemalteca de Educación Superior por el arbitraje.

\section{Referencias}

Hoyos, A. y Gallego, T. (2017). Desarrollo de habilidades de comprensión lectora en niños y niñas de la básica primaria. Revista Virtual Universidad Católica del Norte, 51, 26. Recuperado de https://www.redalyc.org/ pdf/1942/194252398003.pdf

Pimienta, J. (2012) Estrategias de enseñanza aprendizaje. Pearson recuperado de http://prepajocotepec.sems. udg.mx/sites/default/files/estrategias_pimiento_0.pdf

Roncal, F y Montepeque, S. (2011) Aprender de Forma Comprensiva y Critica. USAID:Saqiltzij recuperado de https://pdf.usaid.gov/pdf_docs/pnaea925.pdf

Solé, I. (1998). Estrategias de Lectura (3a.ed.) Grao 


\section{Sobre la autora}

\section{Juana Isabel Rodríguez Bach}

Es licenciada en la enseñanza del Idioma Español y Literatura, egresada de la Escuela de Formación de Profesores Enseñanza Media-EFPEM de la Universidad de San Carlos de Guatemala. Catedrática de diferentes niveles educativos por más de 20 años, ha participado como ponente en congresos de lectura y escritura a nivel nacional e internacional. Participa constantemente en talleres de: educación, literatura, lectura y escritura. Es estudiante de la Maestría en Docencia Universitaria de la Facultad de Humanidades de la Universidad de San Carlos de Guatemala.

Copyright (c) Juana Isabel Rodríguez Bach

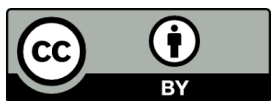

Este texto está protegido por una licencia CreativeCommons 4.0.

Usted es libre para compartir, copiary redistribuir el material en cualquier medio o formato y adaptar el documento, remezclar, transformar y crear a partir del material para cualquier propósito, incluso comercialmente, siempre que cumpla la condición de atribución: usted debe reconocer el crédito de una obra de manera adecuada, proporcionar un enlace a la licencia, e indicar si se han realizado cambios. Puede hacerlo en cualquier forma razonable, pero no de forma tal que sugiera que tiene el apoyo del licenciante o lo recibe por el uso que hace. 